\title{
BASES PARA ENTENDIMENTO DO CROWDSOURCING E SUA APLICAÇÃO EM PROJETOS DE CROWD-DESIGN
}

\author{
DICKIE, Isadora Burmeister. \\ PPGDesign/UFPR \\ isadora.dickie@gmail.com \\ SANTOS, Aguinaldo dos. \\ PPGDesign/UFPR \\ asantos@ufpr.br
}

\begin{abstract}
Resumo: Este artigo apresenta um estudo teórico sobre a aplicabilidade do crowdsourcing em projetos de crowd-design. Utilizou-se como método a revisão bibliográfica sistemática (RBS) tendo como base portais de indexação de documentos em formato de artigos e capítulos de livros. Apresenta-se, portanto, além do protocolo utilizado na realização da RBS, os conceitos de crowdsourcing e os fins diversos para os quais vem aplicado. O que se percebe é que a RBS, quando bem planejada, mostra-se eficaz na busca de artigos que abordam os temas de interesse. Com relação aos trabalhos analisados, nota-se que trata-se de um tema recente e que há muitas lacunas com relação à sua forma de aplicação, inclusive no que se refere ao desenvolvimento e produtos.
\end{abstract}

Palavras-chave: revisão bibliográfica sistemática, crowd-design, crowdsourcing

\begin{abstract}
This paper presents a theoretical study on the applicability of crowdsourcing in crowd-design projects. The method used was the systematic literature review (SLR) based on indexing documents portals in articles and book chapters formats. It presents, therefore, beyond the protocol used in the making of SLR, the concepts of crowdsourcing and the various purposes for which it is applied. What is noticeable is that the SLR, when well planned, is effective in finding articles that cover topics of interest. Regarding the studies analyzed, we note that this is a recent issue and that there are many shortcomings with regard to its application form, including for the product development.
\end{abstract}

Keywords: systematic literature review, crowd-design, crowdsourcing

\section{INTRODUÇÃO}

A facilidade do compartilhamento instantâneo de informações entre multidões, proporcionada principalmente pela internet, está afetando não somente a maneira das 
pessoas se comportarem e se relacionarem, como também a maneira de se projetar em design. Manzini et al. (2004) já entendiam a atividade projetual de Design como sendo um processo que permite: (1) ter uma ideia daquilo que se quer como solução; (2) conhecer ou buscar os recursos disponíveis e (3) implementar a estratégia adequada para alcançar os resultados. Tendo em vista que estas habilidades não pertencem apenas aos designers com formação universitária, para Freire e Damásio (2009), a atividade projetual já saiu dos escritórios de desenvolvimento de produtos para se tornar um processo difuso.

Algumas práticas já demonstram que a abertura dos projetos para a multidão faz com que usuários e consumidores tenham voz ativa nas decisões do processo de desenvolvimento de produtos e serviços. Isso contribui de maneira significativa para a inovação em empresas dos mais diversos setores. O caso da empresa Procter \& Gamble (P\&C), citado por Albors et al. (2008) e Enkel et al. (2009), é um dos exemplos onde os processos internos de inovação em produtos foram abertos através da prática do crowdsourcing. Esta prática viabilizou uma mudança na política de propriedade intelectual da empresa, passando a patente de seus produtos para terceiros, ou seja, para os participantes dos processos abertos de desenvolvimento de produtos. Outra mudança percebida pela empresa foi o fato de que, com a abertura dos processos para a multidão, a empresa aumentou em $50 \%$ a taxa de satisfação com os produtos.

Neste contexto, o crowd-design pode ser entendido como uma modalidade emergente de sistemas de projeto e produção que utiliza os conhecimentos e recursos disponíveis na multidão, geralmente através da internet, com o propósito de resolver problemas e/ou criar conteúdo. Sua realização pode ocorrer de forma voluntária ou remunerada. Assim, esse artigo apresenta um estudo teórico sobre a aplicabilidade do crowdsourcing em projetos de crowd-design. O que se percebe, com base na literatura consultada, é que as mudanças em processos de desenvolvimento de produtos e modelos de negócios estão se direcionando para o "crowd" (multidão, em tradução livre do inglês) devido à economia de tempo e dinheiro que estes processos proporcionam. Também, porque envolver muitos usuários na busca por soluções podem aumentar as chances de êxito. Sendo assim, este estudo busca esclarecer como o crowdsourcing pode ser aplicado na prática do crowd-design.

Para a realização desse estudo, utilizou-se o método da revisão bibliográfica sistemática (RBS), cujo protocolo é detalhado na subseção 2.1. O portal de consulta foi o Periódico CAPES ${ }^{1}$, já que inclui em seu sistema de busca artigos de outros portais, como SCOPUS ${ }^{2}$ e Science Direct ${ }^{3}$, por exemplo.

Como resultado, este artigo traz, além do protocolo seguido para a realização da RBS, os principais conceitos de crowdsourcing e qual a natureza dos projetos que utilizam esta prática. Por fim, discute-se a aplicabilidade do crowdsourcing em projetos de crowd-design, onde, apesar dos estudos já apontarem para alguns cuidados, ainda há muitas lacunas que demandam novos estudos.

\section{METODOLOGIA E DESENVOLVIMENTO}

\footnotetext{
1 www.periodicos.capes.gov.br/

2 www.scopus.com/scopus/home.url

3 www.sciencedirect.com/
} 
A RBS consistiu na busca de artigos do Portal de Periódicos CAPES, associando as palavras crowdsourcing e design. A realização da RBS seguiu um cronograma, baseando-se nas etapas propostas por Conforto et al. (2011), ilustrada na figura 1.

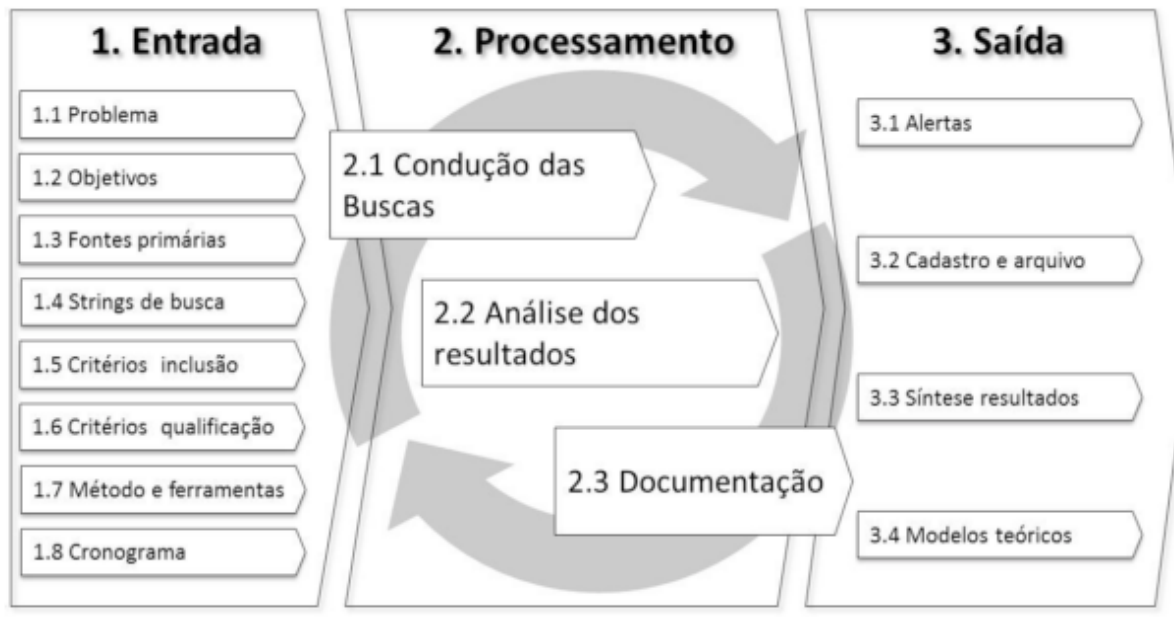

Figura 1 - Etapas para a realização de uma RBS

Fonte: Conforto et al. (2011)

Estas etapas foram executadas de acordo com o cronograma apresentado no quadro 1.

Quadro 1 - Cronograma da RBS

\begin{tabular}{|c|l|c|c|}
\hline $\begin{array}{c}\text { Etapas da RBS segundo } \\
\text { Conforto et al. (2011) }\end{array}$ & \multicolumn{1}{|c|}{ Procedimentos realizados } & $\begin{array}{c}\text { MARÇO } \\
\mathbf{2 0 1 4}\end{array}$ & $\begin{array}{c}\text { ABRIL } \\
\mathbf{2 0 1 4}\end{array}$ \\
\hline \multirow{2}{*}{ ENTRADA } & Entendimento teórico a respeito da RBS & $\mathrm{X}$ & \\
\cline { 2 - 4 } & Elaboração do Protocolo da RBS (Entrada) & $\mathrm{X}$ & $\mathrm{X}$ \\
\hline \multirow{3}{*}{ PROCESSAMENTO } & $\begin{array}{l}\text { Pesquisa nas bases de dados de acordo com os } \\
\text { strings de busca (Processamento) }\end{array}$ & $\mathrm{X}$ & $\mathrm{X}$ \\
\cline { 2 - 4 } & Realização da Seleção 1 (Processamento) & $\mathrm{X}$ \\
\cline { 2 - 4 } & Realização da Seleção 2 (Processamento) & $\mathrm{X}$ \\
\cline { 2 - 4 } & Leitura Completa dos Artigos (Processamento) & $\mathrm{X}$ \\
\hline SAÍDA & $\begin{array}{l}\text { Organização dos dados e informações nas } \\
\text { ferramentas (Saída) }\end{array}$ & & \\
\hline
\end{tabular}

Fonte: Elaborado pelos autores.

\subsection{Protocolo da revisão bibliográfica sistemática}

De acordo com Conforto et al. (2011, p. 6) "a definição do problema é o ponto de partida da revisão bibliográfica sistemática." Assim, definiu-se como problema a seguinte questão: como o crowdsourcing pode ser aplicado ao crowd-design? A busca pela resposta, portanto, seguiu objetivos principais e secundários, que foram "a base para a análise dos artigos encontrados nas buscas." (CONFORTO et al., 2011, p. 6). Objetivo principal: Elucidar de que maneira o crowdsourcing pode ser aplicado à prática do crowd-design. Objetivos Secundários: (a) elucidar os conceitos de crowdsourcing; (b) relacionar os periódicos que mais publicam sobre o tema; (c) relacionar a quantidade de publicações por ano; (d) evidenciar os principais contextos e objetivos de aplicação do crowdsourcing.

A escolha das fontes primárias se deu de acordo com a realização de uma pesquisa bibliográfica preliminar, ou seja, sem o rigor de uma revisão sistemática 
(CONFORTO et al., 2011). A partir desta investigação inicial, foi possível definir as palavras-chave da pesquisa, ou os strings de busca. Os strings de busca, ou seja, os termos utilizados no preenchimento dos campos específicos nas plataformas de base de dados, foram "crowdsourcing" e "design". A aplicação desses strings de busca se deu de duas maneiras: a primeira busca utilizou as palavras como chave de busca específica, e a segunda busca utilizou as palavras combinadas, como por exemplo, "crowdsourcing" + "design".

Os critérios de inclusão utilizados, dentre os disponíveis na plataforma Portal de Periódicos da CAPES, foram: "somente artigos"; "artigos no idioma inglês"; "artigos revisados por pares" e "recorte de tempo: artigos publicados nos últimos 10 anos". Para a definição dos critérios de qualificação, levou-se em consideração os objetivos apresentados anteriormente. Assim, definiu-se que interessariam para o estudo artigos que, além de definir e conceituar os termos citados, também trouxessem exemplos práticos e resultados destas aplicações ou análises.

Já o método utilizado para a RBS, foi: elaboração do protocolo; acesso à base de dados do Portal de Periódicos da CAPES durante as duas primeiras semanas do mês de março do corrente ano para seleção dos artigos a partir da leitura do título e resumo (Seleção 1). Após as buscas, foi realizada a leitura completa dos artigos (Seleção 2). As ferramentas selecionadas para serem utilizadas na etapa de processamento e saída de informações foram, respectivamente, o software Meledey ${ }^{4}$ e um software de edição de planilhas. Por fim, seguiu-se o cronograma já apresentado na tabela 1.

\subsection{Dados acerca da Revisão Bibliográfica Sistemática}

A tabela 1 apresenta a quantidade de artigos encontrados pelo Sistema de Busca do Portal de Periódico CAPES para os strings de busca utilizados junto aos critérios de inclusão.

Tabela 1 - Quantidade de Artigos encontrados pelo Sistema de Busca do Portal de Periódico CAPES, de acordo com os strings de busca

\begin{tabular}{|c|c|c|}
\hline Strings de Busca Utilizados & Critérios de Inclusão Acionados & $\begin{array}{l}\text { Número de } \\
\text { Artigos } \\
\text { encontrados } \\
\text { pelo Sistema }\end{array}$ \\
\hline "Crowdsourcing" & $\begin{array}{l}\text { - Somente artigos; } \\
\text { - Artigos no idioma inglês; } \\
\text { - } \text { Artigos revisados por pares; } \\
\text { - Recorte de tempo: artigos publicados } \\
\text { nos últimos } 10 \text { anos. }\end{array}$ & $\begin{array}{c}1.195 \\
\text { (mil cento e } \\
\text { noventa e cinco } \\
\text { artigos) }\end{array}$ \\
\hline "Crowdsourcing" + "Design" & $\begin{array}{l}\text { - Somente artigos; } \\
\text { - Artigos no idioma inglês; } \\
\text { - } \text { Artigos revisados por pares; } \\
\text { - Recorte de tempo: artigos publicados }\end{array}$ & $\begin{array}{l}\quad 598 \\
\text { (quinhentos e } \\
\text { noventa e oito } \\
\text { artigos) }\end{array}$ \\
\hline
\end{tabular}

4 Mendeley é um gerenciador de referências livre (grátis) para estudantes e pesquisadores. A partir de sua utilização é possível criar uma biblioteca digital totalmente pesquisável em segundos, citar enquanto escreve, bem como ler e fazer anotações em PDFs em qualquer dispositivo (Disponível em: http://www.mendeley.com/en/2/1/). Acesso em 30/04/2014. 
\begin{tabular}{l|l|l}
\hline & nos últimos 10 anos. & \\
\hline Fonte: Dos autores.
\end{tabular}

O filtro para o string de busca "crowdsourcing", mesmo com os quatro critérios de inclusão acionados, encontrou mais de mil artigos. Desta maneira, restringiu-se a busca adicionando o termo "design". Assim, o número de artigos diminuiu pela metade. Destes, foram selecionados 41 (quarenta e um) artigos para a leitura integral, a partir da leitura do título, resumo e das palavras-chave. A leitura completa dos artigos foi realizada virtualmente, através do software Mendeley. Este software permite destacar partes relevantes do texto e incluir comentários no próprio arquivo. Também, auxilia na elaboração automática da lista de referências. Os conteúdos extraídos de cada artigo foi organizado em um software de edição de planilhas, sendo os principais: (a) nome dos autores; (b) ano da publicação; (c) título do artigo; (d) definição de crowdsourcing; (e) se o artigo citava o Mechanical Turk ${ }^{5}$ como exemplo de plataforma crowdsourcing; (f) assunto ou contexto de aplicação do crowdsourcing; e (g) comentários gerais a respeito da aplicabilidade para o crowd-design.

Estes procedimentos foram fundamentais para: (1) a leitura direcionada dos artigos, pois, já sabia-se o que se estava buscando de informação e (2) a organização dos dados para posterior análise (apresentada no tópico 3).

Com relação aos periódicos que mais publicam sobre o tema, percebe-se que a maioria pertence às áreas de Computação e Tecnologia da Informação. Apesar disso, também foram encontradas publicações em periódicos de áreas relacionadas ao Marketing, Gerenciamento de Negócios, Conhecimento e Processos, bem como da área de Psicologia Social.

De acordo com o critério de inclusão "recorte de tempo: artigos publicados nos últimos 10 anos", a busca trouxe o seguinte resultado: (a) nenhum artigo para anos anteriores a 2008; (b) dois artigos de 2008 a 2009 (sendo um artigo por ano); (c) dois artigos em 2010; (d) seis artigos em 2011; (e) 14 artigos em 2012; (f) 16 artigos em 2013 e (g) dois artigos em 2014 (considerando até o mês de abril). Considerando-se que o termo crowdsourcing apareceu pela primeira vez em 2006, percebe-se, portanto que as discussões sobre os temas desta pesquisa são recentes e vem ganhado representatividade ao longo dos últimos quatro anos: 38 artigos publicados entre 2011 e 2014 (até o mês de abril), comparado aos quatro artigos publicados entre 2008 e 2010.

\section{RESULTADOS E DISCUSSÃO}

\subsection{Definição de Crowdsourcing}

Dos 41 trabalhos que compõem a RBS, 16 referenciam Jeff Howe como autor do termo e apresentam a sua definição. Porém, outros autores apresentam diferenças na definição do mesmo termo. O quadro 2 contém a relação das principais definições de crowdsourcing encontradas e seus respectivos autores. A relação foi organizada em ordem cronológica para, desta forma, observar se houve alteração ao longo dos anos.

\footnotetext{
5 Este dado foi acrescentado a partir da leitura do terceiro artigo, onde percebeu-se que os dois artigos lidos anteriormente também citavam esta plataforma. Ao final da leitura dos artigos, percebeu-se que é a plataforma de crowdsourcing mais citada.
} 
Quadro 2 - Definições de Crowdsorcing encontradas na RBS

\begin{tabular}{|c|c|}
\hline Autor(es)/Data & Definição de crowdsourcing \\
\hline $\begin{array}{l}\text { Howe (2006), também citado por } \\
\text { ENKEL et al. (2009); CORNEY et al. } \\
\text { (2010); BÜCHELER E SIEG (2011); } \\
\text { ALONSO E MIZZARO (2012); } \\
\text { ESTELLES-AROLA E GONZALES- } \\
\text { LADRON-DE-GUEVARA (2012); } \\
\text { ZAHO e ZHU (2012); BAYUS } \\
\text { (2013); BANNERMAN (2013); DAI } \\
\text { et al. (2013); DJELASSI e } \\
\text { DECOOPMAN (2013); } \\
\text { GASSENHEIMER et al. (2013); } \\
\text { GERBER e HUI (2013); GUPTA e } \\
\text { SHARMA (2013); HIRTH et al. } \\
\text { (2013); TUNG e TSENG (2013); } \\
\text { WEEKS e VELTRI (2013) }\end{array}$ & $\begin{array}{l}\text { Refere-se ao ato de uma empresa ou instituição terceirizar [ou } \\
\text { fazer um convite aberto] para uma rede indefinida (e } \\
\text { geralmente grande) de pessoas, um trabalho ou tarefa que, } \\
\text { geralmente, seria realizado pelos seus próprios funcionários. }\end{array}$ \\
\hline $\begin{array}{l}\text { Kleemann et al. (2008, apud } \\
\text { BEHREND et al., 2011) }\end{array}$ & $\begin{array}{l}\text { Refere-se à mobilização intencional para exploração comercial } \\
\text { de ideias criativas e outras formas de trabalho realizadas por } \\
\text { consumidores. }\end{array}$ \\
\hline Corney et al. (2010) & $\begin{array}{l}\text { É uma ferramenta para facilitar a inteligência das máquinas em } \\
\text { uma fábrica baseada no conhecimento. }\end{array}$ \\
\hline Adams (2011) & $\begin{array}{l}\text { Crowdsourcing refere-se a um mecanismo específico que as } \\
\text { empresas usam para se envolverem com os consumidores. } \\
\text { Tarefas como a resolução de problemas e controle de qualidade, } \\
\text { uma vez que foram realizados, quer internamente ou } \\
\text { contratados para os funcionários externos, estão agora } \\
\text { "terceirizadas" para os grupos-alvo específicos, públicos ou } \\
\text { gerais ("a multidão") através da web. }\end{array}$ \\
\hline Wexler (2011) & $\begin{array}{l}\text { Pode ser entendido como o uso de uma entidade focal em uma } \\
\text { multidão entusiasmada ou vagamente ligada para fornecer } \\
\text { soluções para problemas. }\end{array}$ \\
\hline $\begin{array}{l}\text { Behrend et al. (2011, apud } \\
\text { AZZAM E JACOBISON, 2013) }\end{array}$ & $\begin{array}{l}\text { É operacionalmente definido como o recrutamento pago de (...) } \\
\text { uma força de trabalho global independente para o objetivo de } \\
\text { trabalhar em uma tarefa especificamente definida ou um } \\
\text { conjunto de tarefas. }\end{array}$ \\
\hline Bannerman (2013) & $\begin{array}{l}\text { O termo 'crowdsourcing' invoca comparações obscuras com a } \\
\text { terceirização, mas, ao mesmo tempo, é otimista, retratado a } \\
\text { maneira de aproveitar a criatividade das massas de graça, ou } \\
\text { por um preço moderado. }\end{array}$ \\
\hline Djelassi e Decoopman (2013) & $\begin{array}{l}\text { Refere-se à abertura dos processos e modelos de negócio da } \\
\text { empresa para "a multidão" por meio de aplicativos da Web } 2.0 \\
\text { com o objetivo de obter acesso a recursos externos (ideias, } \\
\text { habilidades, conhecimentos, tecnologias, etc.). }\end{array}$ \\
\hline
\end{tabular}

Fonte: Dos autores, baseados nos autores citados no próprio quadro. 
Assim, apesar de não explicitar que o crowdsourcing pode acontecer de maneira que haja a remuneração dos participantes, a definição mais utilizada é a de Howe, de 2006. Apenas a partir de 2011 é que a definição de crowdsourcing aponta para a questão da remuneração (BEHREND et al., 2011, apud AZZAM E JACOBISON, 2013). Todas as definições encontradas, porém, enfatizam a utilização do conhecimento e/ou criatividade da multidão para um determinado propósito.

Estelles-Arola e Gonzales-Ladron-de-Guevara (2012) realizaram um estudo aprofundado a respeito das definições de crowdsourcing e, a partir da análise de casos, chegaram a seguinte definição:

[...] é um tipo de atividade participativa online, na qual pessoas e/ou empresas propõem para um grupo de pessoas de várias áreas do conhecimento, heterogêneo e numeroso, a partir de uma convite aberto, o engajamento voluntário a uma tarefa. A tarefa, de complexidade e modularidade variadas, e da qual a multidão deve participar oferecendo seu trabalho, dinheiro, conhecimento e/ou experiência, sempre implica em benefício mútuo. O usuário irá receber a satisfação para determinada necessidade, seja ela econômica, reconhecimento social, auto estima ou o desenvolvimento de habilidades individuais, enquanto que o demandante da tarefa irá obter e utilizar para seu benefício os resultados que os usuários trouxerem, dependendo do tipo de atividade demandada. (ESTELLESARROLA e GONZALES-LADRON-DE-GUEVARA, 2012, p. 197).

A definição destes autores traz uma síntese do que vem a ser o crowdsourcing, acrescentando que podem haver desdobramentos, como por exemplo, o engajamento a multidão no financiamento de projetos. Segundo Bannerman (2013) e Djelassi e Decoopman (2013), quando isto acontece, o processo é chamado de crowdfunding ${ }^{6}$. Geralmente estes financiamentos ocorrem para a viabilização de projetos culturais. A plataforma kickstarter.com, por exemplo, possui 13 categorias diferentes de projetos financiáveis: arte, história em quadrinhos, dança, moda, comida, jogos, fotografia, edição, tecnologia, teatro, cinema, música, e design, sendo estes três últimos os mais populares entre os usuários (ZHOU, 2012). Outro desdobramento apontado por Djelassi e Decoopman (2013) é o crowdlabor $^{7}$, onde o consumidor fornece o trabalho e executa tarefas que vão das mais simples às mais complexas. A diferença para o crowdsourcing é que o participante é considerado um trabalhador real da empresa (DJELASSI e DECOOPMAN, 2013).

\subsection{Crowdsourcing e sua aplicabilidade ao Crowd-Design}

Para Corney et al. (2010), a aplicacão do crowdsourcing oferece a oportunidade de descobrir efetivas estratégias de resolução à problemas, pois devido à natureza digital da atividade, é possível gravar, observar e avaliar as estratégias de solução à problemas da perspectiva de muitos indivíduos. Essas atividades são executadas por pessoas que não necessariamente se conhecem, mas interagem com a empresa através de ferramentas virtuais e da conexão com a internet. Por isso, inúmeras são as aplicações possíveis, e a natureza dos projetos são diversas. Nesse sentido, no quadro

\footnotetext{
6 São exemplos de plataformas de crowdfunding: kickstarter.com, mymanagercompagny.com, indiegogo.com.

${ }^{7}$ Por exemplo: mobileworks.com, samasource.org e transcribeme.com.
} 
3 há uma relação sobre a natureza das principais abordagens encontradas na literatura consultada.

Quadro 3 - Natureza das principais aplicações de crowdsourcing

\begin{tabular}{|l|l|}
\hline \multicolumn{1}{|c|}{ Objetivo do Projeto } & \multicolumn{1}{|c|}{ Referências } \\
\hline Aprendizagem e colaboração em rede & ALBORS et al., (2009); CORNEY et al. (2010) \\
\hline $\begin{array}{l}\text { Pesquisa e desenvolvimento (científico e não } \\
\text { científico) }\end{array}$ & ENKEL et al. (2009); BÜCHELER e SIEG (2011) \\
\hline Desenvolvimento de produtos & $\begin{array}{l}\text { BRABHAM (2010); DJELASSI e DECOOPMAN } \\
\text { (2013) }\end{array}$ \\
\hline Melhoria de serviços & ADAMS (2011) \\
\hline Pesquisa de opinião & BEHREND et al. (2011) \\
\hline Resolução de problemas & SANSOM (2011) \\
\hline Avaliação de relevância & ALONSO (2012); ALONSO e MIZZARO (2012) \\
\hline
\end{tabular}

Fonte: Dos autores.

Para ilustrar com mais detalhes sobre a natureza das aplicações de crowdsourcing, Zaho e Zhu (2012) utilizaram duas dimensões para classificá-las: contexto e função. O contexto, por sua vez, foi dividido em duas categorias: empresarial e não-empresarial. O primeiro contexto inclui empresas, organizações sem fins lucrativos ou mercados, enquanto que o segundo inclui as organizações sem fins lucrativos ou instituições, tais como bibliotecas públicas, centro de pesquisa e desenvolvimento, governo, etc., onde a participação em massa e a colaboração científica acontecem. Para os autores, o contexto de uma aplicação desempenha um papel importante no sentido de refletir os impactos e significados do crowdsourcing.

Já a dimensão da função representa a parte do produto e/ou do ciclo de vida do serviço que está sendo requisitado através do crowdsourcing (VUKOVIC, 2009 apud ZAHO e ZHU, 2012). Ou autores afirmam que é possível caracterizar as funções do crowdsourcing pela natureza e complexibilidade da tarefa. Tarefas de baixa complexidade geralmente referem-se a tarefas de rotina, como a coleta de dados, classificação e tradução de textos simples. Tarefas de média complexidade geralmente se referem às tarefas criativas, como desenvolvimento de logotipo, fotografia ou publicidade gerada pelo usuário. Já as tarefas de alta complexidade estão relacionadas, principalmente, ao desenvolvimento de produtos (ZAHO e ZHU, 2012). Ainda, para Djelassi e Decoopman (2013), o crowdsourcing pode ser classificado, de acordo com as tarefas, em não criativo e criativo, sendo este último associado ao desenvolvimento de produtos.

Djelassi e Decoopman (2013) abordaram a questão do desenvolvimento de produtos, porém, sob a ótica do Marketing. Como resultado, encontraram as interrelações entre os diferentes componentes deste modelo de negócio e as interações entre empresas e clientes. A principal limitação do estudo citado, porém, é que foram entrevistados apenas os consumidores que já tinham experiência na participação de um processo de crowdsourcing, mas não ficou claro se estes 
participantes eram Designers ou profissionais de áreas afins. O estudo também não esclarece quais os obstáculos e os medos que limitam a participação dos usuários que não são familiarizados com a prática do crowdsourcing. Além disso, as empresas da amostra do estudo foram empresas de bens de consumo, que, segundo os autores, é o setor em que o crowdsourcing é usado com mais frequência. Ainda, na opinião dos autores, valeria a pena estender a pesquisa para outros setores, como o de serviços ou bens de consumo duráveis (DJELASSI e DECOOPMAN, 2013).

Brabham (2010), no entanto, comenta que o envolvimento de não-especialistas na resolução de problemas de design pode trazer soluções de qualidade superior e mais rentáveis para as empresas. O autor acredita, ainda, que o processo de desenvolvimento de soluções a partir do crowdsourcing gera uma riqueza de dados, e que as ideias vencedoras contribuem de forma significativa para a compreensão de como as pessoas resolveriam um determinado problema, podendo ser fonte de inspiração para a inovação. Nesse sentido, o caso da empresa P\&G, citado por Albors et al. (2008), ilustra as vantagens da utilização do crowdsourcing para a inovação: antes da abertura do processo, apenas $10 \%$ da capacidade de inovação da empresa era utilizada. No entanto, os autores não esclarecem como se deu este processo; apenas enfatizam que, para este processo ser sustentável, depende do incentivo e motivação oferecidos aos participantes. Já Enkel et al. (2009) apontam para a ocorrência da desvantagem competitiva em empresas que não aproveitam a oportunidade de inovar incluindo a multidão em seus processos, pois, para os autores, "a maior inovação é baseada em uma recombinação do conhecimento existente, conceitos e tecnologia" (ENKEL et al., 2009, p. 314).

Massanari (2012), por sua vez, analisou cinco plataformas de crowdsourcing com foco em projetos de design gráfico ${ }^{8}$, com o objetivo de esclarecer as mudanças que ocorrem na maneira de se projetar. Constatou que os Designers que participam deste tipo de processo provavelmente recebem uma remuneração muito menor do que os salários, tendo como base os valores de mercado dos Estados Unidos. Talvez porque, segundo a autora, as empresas que demandam este tipo de projeto através das plataformas não tenham conhecimento sobre a importância e o valor que o design possui. Neste mesmo sentido, encontrou-se em Bannerman (2013) a informação de que algumas organizações internacionais de design têm se revelado contra o crowdsourcing. Um exemplo é a organização SPEC NO!, formada por Designers para protestar contra concursos, onde, muitas vezes, os designers apresentam projetos, mas não tem a garantia de pagamento. Esta pode ser, sem dúvida, uma forte desvantagem em se utilizar o crowdsourcing em projetos de crowd-design.

Apesar disso, a quantidade de plataformas de crowdsourcing é crescente. A Plataforma Amazon Mechanical Turk, por exemplo, foi citada pela maioria dos artigos como a mais conhecida e, portanto, referência no quesito plataforma de crowdsourcing para a realização de tarefas de baixa complexidade - de acordo com a classificação de Zhao e Zhu (2012). O quadro 4 traz a lista de outras plataformas, citadas por, pelo menos, dois artigos. 8 Plataformas analisadas por Massanari (2012): 99designs.com; designbyhumans.com; designcrowd.com;
huffingtonpost.com; threadless.com 
Quadro 4 - Plataformas de Crowdsourcing citadas em mais de um artigo

\begin{tabular}{|l|l|l|}
\hline \multicolumn{1}{|c|}{ Plataforma } & \multicolumn{1}{|c|}{ Link } & \multicolumn{1}{c|}{ Referências } \\
\hline Mechanical Turk & www.mturk.com & $\begin{array}{l}\text { ALONSO e MIZZARO (2012); ALONSO (2012) e AZZAM e } \\
\text { JACOBSON (2013) }\end{array}$ \\
\hline 99Designs & www.99designs.com & BANNERMAN (2013) e ZHAO e ZHU (2013) \\
\hline Odesk & www.odesk.com & AZZAM e JACOBSON (2013) e DIN, LAN, WELD (2013) \\
\hline INNOCENTIVE & www.innocentive.com & ALBORS, RAMOS e HERVAS (2008) e BANNERMANS (2013) \\
\hline
\end{tabular}

Fonte: Dos autores.

Ainda, Zaho e Zhu (2012) apontaram como plataformas exclusivas de crowdsourcing para desenvolvimento de produtos: 99Designs, Threadless (www.threadless.com), iStockPhoto (www.istockphoto.com), Mass Mapping (site não encontrado) e People Per Hour (www.peopleperhour.com).

A utilização destas plataformas requer, no entanto, conhecimento sobre o funcionamento do processo, incluindo a fase de preparação. Nesse sentido, Alonso (2012) enfatiza a importância dos estágios iniciais, ou seja, da preparação de uma tarefa: primeiro, deve-se escolher, criteriosamente, a plataforma de crowdsourcing onde a tarefa será disponibilizada; segundo, deve-se testar a realização da tarefa com uma equipe interna, para ter certeza de que as informações estão claras, e que a tarefa pode ser realizada inclusive por não especialistas.

Já Brabham (2010) aponta nove princípios norteadores para a elaboração da tarefa: (1) definir claramente o problema e as soluções que são esperadas; (2) determinar o nível de comprometimento da empresa com os resultados obtidos, no sentido de explicitar o tipo de solução que será produzida e porquê; (3) entender o que motiva a participação dos usuários; (4) investir em um site que é utilizável, interessante e bem concebido; (5) ter um plano promocional e um plano para o crescimento da comunidade; (6) ser honesto, transparente e ágil; (7) não controlar o comportamento da multidão, pois ela não está sendo consultada para realizar os objetivos organizacionais e sim convidada a se envolver no processo de desenvolvimento de produtos; (8) reconhecer os usuários, entregando-lhes as premiações que lhe forem prometidas; (9) avaliar o projeto sob vários ângulos, solicitando sempre o feedback dos participantes. Seguindo estes princípios, o autor acredita ser possível elaborar um projeto de crowdsourcing que seja eficaz.

\section{CONSIDERAÇÕES FINAIS}

Para contribuir com o entendimento das mudanças que estão ocorrendo na maneira de se projetar em design, este artigo apresentou um estudo para elucidar a aplicabilidade do crowdsourcing em projetos de crowd-design. Como método de investigação teórica, a RBS mostrou-se eficaz, sobretudo na organização da pesquisa. A principal vantagem foi iniciar a pesquisa a partir da elaboração de um protocolo, que guiou, principalmente, os primeiros estágios e sistematizou a busca de artigos.

Assim, pode-se entender que o crowdsourcing é uma prática recente e que vem sendo investigada por várias áreas do conhecimento. Devido à redução de tempo e custo para a realização de tarefas que possuem diferentes fins e graus de 
complexidade, esta prática é crescente entre as empresas, sendo relacionada principalmente à inovação. Esta pode estar associada tanto à abertura do processo, como aos próprios resultados obtidos.

Apesar das investigações científicas sobre o assunto terem se intensificado somente a partir dos anos 2010, no que diz respeito a projetos de crowd-design, muitas ainda são as lacunas. Faltam, por exemplo, investigações de casos específicos sobre projetos de desenvolvimento de produtos, onde seria possível esclarecer as dúvidas a respeito da motivação dos participantes, do teor das tarefas (clareza das informações, grau de complexidade, tempo disponível para a execução, dentre outros) e das respectivas remunerações. Talvez, por este assunto não ser muito explorado em estudos científicos, o que se encontrou como desvantagem foi o fato de algumas entidades de design perceberem esta prática como especulativa. Como ainda não estão claros os procedimentos para a aplicação do crowdsourcing para o crowd-design, não se apresenta claro, também, qual é o papel do Designer neste tipo de processo.

\section{AGRADECIMENTOS}

Os autores agradecem o apoio financeiro da CAPES (Coordenação de Aperfeiçoamento de Pessoal de Nível Superior) pela bolsa de doutorado demanda social.

\section{REFERÊNCIAS}

ADAMS, S. Sourcing the crowd for health services improvement: The reflexive patient and "share-your-experience" websites. In: Social science \& medicine, v. 72, n. 7, p. 1069-1076, 2011.

ALBORS, J; RAMOS, J. C.; HERVAS, J. L. New learning network paradigms: Communities of objectives, crowdsourcing, wikis and open source. In: International Journal of Information Management, v. 28, n. 3, p. 194-202, 2008.

ALONSO, O.; MIZZARO, S. Using crowdsourcing for TREC relevance assessment. In: Information Processing \& Management, v. 48, n. 6, p. 1053-1066, 2012.

AZZAM, T.; JACOBSON, M. Finding a Comparison Group: Is Online Crowdsourcing a Viable Option? In: American Journal of Evaluation, v. 34, n. 3, p.372-384, 2013.

BANNERMAN, S. Crowdfunding Culture. In: Journal of Mobile Media, v. 07, n. 01, p. 130, 2013.

BAYUS, B. L. Crowdsourcing New Product Ideas over Time: An Analysis of the Dell IdeaStorm Community. In: Management Science, v. 59, n. 01, p. 226-244, 2013.

BEHREND, T. S.; SHAREK, D. J.; MEADE, A. W. The viability of crowdsourcing for survey research. In: Behavior research methods, v. 43, n. 3, p. 800-813, 2011.

BRABHAM, D. C. CROWDSOURCING AS A MODEL FOR PROBLEM SOLVING: LEVERAGING THE COLLECTIVE INTELLIGENCE OF ONLINE COMMUNITIES FOR PUBLIC GOOD. Tese. Department of Communication The University of Utah, 2010.

BUCHELER, T.; SIEG, J. Understanding Science 2.0: Crowdsourcing and Open Innovation in the Scientific Method. In: Procedia Computer Science, v. 7, p. 327-329, 2011.

CONFORTO, E. C.; AMARAL, D. C.; SILVA, S. L. da. Roteiro para revisão bibliográfica sistemática: aplicação no desenvolvimento de produtos e gerenciamento de projetos. 8을 Congresso Brasileiro de Gestão de Desenvolvimento de Produto. Anais do 8ㅇ CBGDP, 2011. Porto Alegre, RS. 
CORNEY, J. R.; TORRES-SANCHÉS, C.; JAGADEESAN, A. P.; YAN, X. T.; REGLI, W. C.; MEDELLIN, H. Putting the crowd to work in a knowledge-based factory. In: Advanced Engineering Informatics, v. 24, n. 3, p. 243-250, 2010.

DAI, P.; LIN, C. H.; WELD, D. S. POMDP-based control of workflows for crowdsourcing. In: Artificial Intelligence, v. 202, p. 52-85, 2013.

DJELASSI, S.; DECOOPMAN, I. Customers' participation in product development through crowdsourcing: Issues and implications. In: Industrial Marketing Management, v. 42, n. 5, p. 683-692, 2013.

ESTELLES-AROLAS, E.; GONZALEZ-LADRON-DE-GUEVARA, F. Towards an integrated crowdsourcing definition. In: Journal of Information Science, v. 38, n. 2, p. 189-200, 2012.

ENKEL, E.; GASSMANN, O.; CHESBROUGH, H. Open R\&D and open innovation: exploring the phenomenon. In: R\&D Management, v. 39, n. 4, p. 311-316, 2009.

FREIRE, K. de M.; DAMAZIO, V. Design de serviços: conceitos e reflexões sobre o futuro da disciplina. $9^{\circ}$ Congresso Brasileiro de Pesquisa e Desenvolvimento em Design. In: Anais do 9o P\&D Design, 2010. São Paulo, SP.

GASSENHEIMER, J. B.; SIGUAW, J. A.; HUNTER, G. L. Exploring motivations and the capacity for business crowdsourcing. In: AMS Review, v. 3, n. 4, p. 205-216, 2013.

GERBER, E. M.; HUI, J. Crowdfunding: Motivations and Deterrents for Participation. In: ACM Trans. Comput.-Hum. Interact. v. 20, n. 6, Article 34 (December 2013), 32 pages. 2013.

GUPTA, D. K.; SHARMA, V. Exploring crowdsourcing: a viable solution towards achieving rapid and qualitative tasks. In: Library Hi Tech News, v. 30, n. 2, p. 14-20, 2013.

HIRTH, M.; HOSSFEL, T.; TRAN-GIA, P. Analyzing costs and accuracy of validation mechanisms for crowdsourcing platforms. In: Mathematical and Computer Modelling, v. 57, n. 11-12, p. 2918-2932, 2013.

MANZINI, E.; COLLINA, L.; EVANS, S. Solution oriented partnership: How to Design industrialised sustainable solutions. In: European Comission Growth Programme. Cranfield: Cranfield University, 2004.

MASSANARI, A. L. DIY design: how crowdsourcing sites are challenging traditional graphc design pratice. In: First Monday, v. 17, n. 10, p. 1-16, 2012.

SANSOM, C. The power of many. Nature Publishing Group, v. 29, n. 3, p. 201-203, 2011.

TUNG, Y.; TSENG, S. A novel approach to collaborative testing in a crowdsourcing environment. In: Journal of Systems and Software, v. 86, p. 2143-2153, 2013.

WEEKS, M.; VELTRI, F. N. Virtual Communities as Narrative Networks: Developing a Model of Knowledge Creation for Crowdsourcing Environments. International Journal of Knowledge Management, v. 9, n. 1, p. 21-41, 2013.

WEXLER, M. N. Reconfiguring the sociology of the crowd: exploring crowdsourcing. In: International Journal of Sociology and Social Policy, v. 31, n. 1/2, p. 6-20, 2011.

ZHAO, Y.; ZHU, Q. Evaluation on crowdsourcing research: Current status and future direction. In: Information Systems Frontiers, 2012. Disponível em: <http://link.springer.com/10.1007/s10796-012-9350-4>. Acesso em: 27/3/2014.

ZHOU, J. Crowdsourcing: A New Revolution for Start-ups. Trabalho de Conclusão de Curso. Leonard N. Stern School of Business. 2012. 